FORMATLON Formation emploi

Revue française de sciences sociales

150 | avril-juin 2020

Former aux "petits" métiers : regards internationaux

\title{
Formation professionnelle en Suisse romande : l'impact des représentations des enseignant·e's sur les inégalités genrées
}

Vocational education and training in French-speaking Switzerland: the impact

of teacher representations on gender inequalities

Die Berufsausbildung in der Westschweiz : die Auswirkungen der

Lehrervertretungen auf die Ungleichheiten zwischen den Geschlechtern

Formación profesional en la Suiza francófona : el impacto de las

representaciones de las/os docentes en las desigualdades de género

\section{Matilde Wenger et Farinaz Fassa}

\section{OpenEdition}

Journals

Édition électronique

URL : https://journals.openedition.org/formationemploi/8203

DOI : 10.4000/formationemploi.8203

ISSN : 2107-0946

Éditeur

La Documentation française

Édition imprimée

Date de publication : 8 juillet 2020

Pagination : $97-121$

ISSN : 0759-6340

Référence électronique

Matilde Wenger et Farinaz Fassa, « Formation professionnelle en Suisse romande : l'impact des représentations des enseignant·e.s sur les inégalités genrées », Formation emploi [En ligne], 150 | avriljuin 2020, mis en ligne le 02 janvier 2022, consulté le 07 janvier 2022. URL : http://

journals.openedition.org/formationemploi/8203 ; DOI : https://doi.org/10.4000/formationemploi.8203

(c) Tous droits réservés 


\section{Formation professionnelle en Suisse romande : l'impact des représentations des enseignant·e·s sur les inégalités genrées}

MATILDE Wenger Doctorante à l'Institut fédéral des hautes études en formation professionnelle (IFFP), Suisse

FARINAZ FASSA Professeure ordinaire à I'Université de Lausanne, Suisse. Elle est affiliée à I'Institut des sciences sociales ainsi qu'au Centre en études genre

Résumé

Formation professionnelle en Suisse romande : l'impact des représentations des enseignant·e.s sur les inégalités genrées

Une étude effectuée auprès d'enseignant.e.s de la formation professionnelle initiale (FPI), en Suisse romande, a permis de mettre en avant le rôle controversé de ces agents de socialisation face à l'égalité des sexes. Les réponses de près de 300 enseignant.e.s à des questions concernant les échelles de sexisme moderne et traditionnel ont permis de révéler des différences statistiquement significatives face aux problématiques de genre. Certains groupes du corps enseignant acceptent - et probablement véhiculent - encore en partie des représentations de genre, transmettant une socialisation différentielle des sexes au monde professionnel.

Mots clés : enseignement technique-professionnel, apprentissage, genre, division sexuelle du travail, division sexuelle de la formation, enseignant, socialisation, représentation de la formation, Suisse

Abstract

Vocational education and training in French-speaking Switzerland: the impact of teacher representations on gender inequalities

A study conducted among teachers of initial vocational education and training (IVET) in French-speaking Switzerland highlighted the controversial role of these socialization agents in addressing gender equality. The responses of nearly 300 teachers to questions about modern and traditional sexism scales highlighted statistically significant differences in gender issues. Some groups of teachers still accept - and probably partly transmit - 
gender representations, transferring a differential socialization of the sexes to the professional world.

Keywords: technical \& vocational education, apprenticeship, gender, gender based division of labour, sexual division of training, teacher, socialisation, perception of training, Switzerland

Journal of Economic Literature: I 21, J 24, J 70

Traduction : auteures.

\section{Introduction}

Être apprenti.e en formation professionnelle duale présume d'être confronté.e, d'une part, au marché du travail et, d'autre part, au contexte scolaire. En effet, l'apprentissage dit dual, tel qu'il est organisé en Suisse, repose sur l'alternance hebdomadaire entre le travail dans le cadre de l'entreprise et la formation professionnelle théorique qui se déroule en école professionnelle (selon les métiers, les apprenti·e.s fréquentent les établissements professionnels un ou deux jours par semaine).

Une telle réalité comporte plusieurs enjeux en termes d'apprentissages, non seulement des compétences liées au métier, mais aussi des attitudes et croyances transmises par les personnes qui forment ces apprenti.e.s. C'est ainsi que ces jeunes apprennent en parallèle les règles d'un métier, ses savoirs et savoir-faire, mais aussi celles du système de genre en raison de la division sexuée existante dans le métier lui-même et dans les pratiques et discours des agents de socialisation que sont les formateurs et formatrices en entreprise et les membres du corps enseignant des écoles professionnelles.

La répartition très sexuée dans les métiers, conjuguée au manque de main-d'œuvre dans certains métiers techniques ont attiré l'attention des autorités politiques sur l'égalité entre les sexes, suite à la crise des places d'apprentissage des années 1990. Depuis le debut des années 2000, plusieurs campagnes ont vu je jour, visant à ouvrir l'horizon professionnel des deux sexes et à lutter contre la ségrégation horizontale ${ }^{1}$ qui reste extrêmement nette en Suisse. L'initiative la plus connue d'entre elles, Futur en tous genres, se déroule à l'échelle nationale et prend, depuis 2001, la forme d'une journée annuelle. Elle permet prioritairement aux enfants de découvrir le métier exercé par un proche de l'autre sexe, mais aussi de sensibiliser les enseignant.e.s aux questions d'inégalités de genre (Fassa \& Naef, 2015).

1. La ségrégation horizontale suit le principe de division : certains métiers ne sont exercés quasiment que par des hommes, alors que d'autres sont réservés aux femmes; la ségrégation verticale suit quant à elle le principe de hiérarchie : dans une même profession, les hommes occupent plus souvent des postes hiérarchiquement supérieurs par rapport aux femmes, ce qui correspond à une distribution inégale des deux sexes dans les différents niveaux de la hiérarchie (Gianettoni \& al., 2015). 
La recherche a en effet révélé que ces personnes sont des agents de socialisation importants. Mosconi $(1999,2001,2004)$ a en outre insisté sur le fait que toute inaction en ce domaine participe de fait au maintien d'un ordre inégal. Or, les enseignant.e.s, comme les formateurs et formatrices, véhiculent - plus ou moins consciemment - des stéréotypes et représentations qui leur sont propres. Si les formateurs et formatrices en entreprise et le contexte professionnel dans lequel les apprenti.e.s apprennent un métier jouent un rôle fondamental (Lamamra, 2016), celui des enseignant.e.s des écoles professionnelles n'est pas à négliger. Il reste notamment à préciser quelles sont leurs croyances à propos des relations entre les femmes et les hommes et de la place de ces deux classes de sexe dans le monde social.

Ce constat rend particulièrement intéressantes les analyses qui ont été effectuées sur les réponses faites aux échelles de sexisme, traditionnel et moderne ${ }^{2}$ (Swim, Aikin, Hall, \& Hunter, 1995), par les enseignant·e.s interrogé.e.s dans le cadre de l'enquête PROFID ; enquête qui portait sur les identités professionnelles des enseignant.e.s de la formation professionnelle vaudoise (Fassa, Dubois, Wenger \& Ceppi, 2019). Ces analyses ont mis en évidence des différences nettes, tout d'abord entre les deux échelles de sexisme, mais aussi selon le sexe, le type de branche enseignée et le statut professionnel des différents groupes de ce corps enseignant.

Ainsi, en partant d'une présentation du système de formation professionnelle helvétique et de ses enjeux dans une approche genrée (partie I), le focus sera mis ici sur des personnes clés de la dimension scolaire de cette formation, les enseignant.e.s. La deuxième partie présentera les résultats de l'étude, en montrant notamment que les enseignant.e.s en formation professionnelle conçoivent différemment la place des femmes et des hommes dans les diverses sphères de vie. En ce sens, les femmes et les hommes qui enseignent en formation professionnelle initiale (FPI), de même que les personnes ayant des formations différentes et jouissant de statuts eux aussi diversifiés ne se positionnent pas de façon similaire lorsqu'il est question de soutien à l'égalité. La dernière partie de l'article sera consacrée à la discussion de ces résultats et à une tentative d'explication de ces constats. Elle permettra de conclure sur les conséquences des représentations des enseignant.e.s de la FPI en termes de socialisation de genre.

2. Le « sexisme traditionnel » concerne les stéréotypes qui envisagent les femmes comme moins compétentes que les hommes, alors que le "sexisme moderne " - plus subtil que le sexisme traditionnel - se définit à la fois par le déni de la persistance de la discrimination sexiste et par l'opposition aux revendications et aux politiques d'égalité. 


\section{Le système de formation professionnelle helvétique face aux défis de l'égalité}

Cette partie présentera la situation de la formation professionnelle suisse en mettant notamment en évidence ses dimensions genrées. Pour cela, nous indiquerons, tout d'abord, quelle est la place de cette formation face à d'autres alternatives. Nous examinerons ensuite comment ce système favorise des choix professionnels sexués, ce qui nous mènera à conclure cette partie sur le rôle central du corps enseignant en formation professionnelle en tant qu'agent de socialisation.

\subsection{Le système de formation professionnelle suisse : un modèle à suivre?}

En Suisse, la formation professionnelle représente le choix privilégié par deux jeunes sur trois au moment de la sortie de l'école obligatoire (Office Fédéral de la Statistique [OFS], 2017). Bien qu'extrêmement développé, puisqu'il concerne une large majorité des jeunes, le système de formation professionnelle initiale suisse présente aujourd'hui quelques faiblesses, selon l'OCDE (Organisation de coopération et de développement économiques ; Fazekas \& Field, 2013). Il repose sur l'organisation des métiers euxmêmes et ne semble pas toujours susceptible de favoriser les reconversions professionnelles ultérieures en raison d'un défaut de formation générale approfondie. L'accent étant en effet plutôt mis sur une socialisation très spécifique aux métiers, notamment grâce à une insertion précoce dans le monde du travail, permise par la formation en alternance. Pouvant théoriquement intervenir dès la fin de l'école obligatoire (15 ans), cette entrée précoce dans le monde du travail favorise une insertion professionnelle future plus aisée que dans d'autres pays européens (Fazekas \& Field, op. cit.).

Or, l'entrée dans le monde du travail est inscrite dans un contexte où le choix de suivre une formation professionnelle initiale (FPI) reste marqué par la reproduction des inégalités sociales (Falcon, 2016) ; en effet, les enfants issus de familles universitaires ont 1,5 fois plus de chances d'intégrer une haute école que les enfants issus de familles non-universitaires (Centre suisse de coordination pour la recherche en éducation [CSRE], 2014).

Notons également que la FPI est surtout fréquentée par des garçons (Fassa, 2016), ces derniers empruntant en outre plus volontiers la voie de l'apprentissage dual. Ainsi, la proportion de filles qui choisissent la voie de la formation professionnelle est nettement plus faible que celle des garçons $(57.7 \%$ contre $74.2 \%$ dans l'année 2013/2014 - Fassa, op. cit.) et, de manière générale, la mixité dans les métiers reste rare (Borkowsky, 2000 ; Fassa, op. cit. ; Lamamra, op. cit.), une segmentation horizontale très nette persistant à marquer les champs professionnels dans lesquels s'engagent les jeunes. En outre, le nombre de métiers qui attire les filles est plus limité que celui 
qui s'offre aux garçons (en 2014, $60 \%$ des filles en FPI étaient réunies dans vingt formations - alors que plus de 230 formations sont proposées en Suisse - Lamamra, Fassa, \& Chaponnière, 2014); ce qui destine les filles à surtout travailler dans les secteurs des professions sociales, de la santé, de l'éducation et des services, alors que les garçons suivent des formations industrielles ou techniques qui se déroulent très majoritairement en alternant pratique du métier en entreprise et cours théoriques en école.

Ajoutons par ailleurs qu'il est possible de se former à un métier par la fréquentation d'une école professionnelle à plein temps, ce choix dépendant des métiers et corollairement du sexe (Buchs \& Müller, 2016). C’est notamment le cas pour certains des métiers techniques et surtout pour ceux qui préparent aux soins aux personnes. La formation pratique est dans ce cas acquise par des stages ultérieurs et des cours donnés dans les écoles par des enseignant·e.s issu.e.s des métiers étudiés et dispensant les cours de connaissances théoriques, mais également pratiques. Ces divers éléments font écho au constat dressé par Vouillot (2007) d'orientations scolaires et professionnelles sexuées qui seraient, selon Gianettoni, Carvalho \& al. (2015), notamment liées à des aspirations de carrière et de formation différenciées entre les filles et les garçons en fin de scolarité obligatoire.

\subsection{Des éducations et carrières professionnelles sexuellement différenciées}

La question de l'(in)égalité entre filles et garçons dans l'éducation et la formation en Suisse est un sujet qui suscite de l'intérêt dans le monde politique, mais aussi dans celui de la recherche. Les travaux réalisés en sociologie de l'éducation et de la formation, comme ceux issus des études de genre (Fassa, Fueger, Lamamra, Chaponnière \& Ollagnier, 2010 ; Fassa, Rolle \& Storari, 2014 ; Flamigni \& Pfister Giauque, 2014 ; Gianettoni \& Guilley, 2016) montrent que l'égalité formelle a été atteinte - l'accès à toutes les filières de l'enseignement et de la formation étant en effet garanti pour les filles et les garçons depuis plusieurs décennies (Fassa, op. cit.).

Ils insistent toutefois aussi sur le fait que des formes d'inégalités persistent tout au long de la scolarité, mais également lors de la formation professionnelle et de l'insertion dans le monde du travail (Lamamra, op. cit.), nécessitant encore des " réajustements". Ces inégalités se traduisent par les ségrégations, horizontale et verticale, phénomènes qui se manifestent dans la structure du marché du travail de plusieurs pays, dont la Suisse (Heiniger \& Imdorf, 2018). Ces formes de division et de hiérarchisation professionnelle établissent à leur tour une séparation sexuée des métiers (Fassa, op. cit.), à laquelle les jeunes qui démarrent une formation professionnelle n'échappent pas. 
Le monde du travail se trouve ainsi être le produit d'une division sexuée de l'orientation scolaire et professionnelle ${ }^{3}$ (Vouillot, op. cit.) et d'aspirations professionnelles très sexuées (Guilley \& al., 2014 ; Gianettoni \& al., 2015 ; Gianettoni \& Guilley, op. cit.). Lamamra \& al. (2014) décrivent par ailleurs les processus qui se déroulent pendant la formation professionnelle comme des processus opérant "une socialisation de genre " "au travers de la division sexuelle du travail" (p. 11). Cela signifie de fait que les jeunes apprennent un métier (en termes de savoirs et savoir-faire, mais aussi de règles de socialisation plus ou moins implicites), mais sont aussi socialisé.e.s au système de genre se manifestant souvent par des formes de hiérarchisation et de division sexuées des tâches dans le métier lui-même.

\subsection{Le rôle des enseignant·e.s comme agents de socialisation}

La littérature sur les inégalités, leurs sources et les éventuelles corrections qu'on peut y apporter rélève par ailleurs que l'influence et le rôle des enseignant.e.s sont considérables (Bressoux, 1994, 2009). Bressoux (1994) parle à ce propos d' "effet-maître " et montre que cet effet participe largement de la construction des inégalités de résultats entre les élèves. Ainsi, le type et la longueur de la formation (Fassa, 2014), l'ancienneté (Bressoux \& Lima, 2011) et les pratiques pédagogiques (Duru-Bellat \& LeroyAudouin, 1990) influent nettement sur les résultats des élèves.

De plus, on doit ajouter les travaux qui portent sur les jugements professoraux : les images que les enseignant.e.s se font des élèves ont tout à voir avec leur propre trajectoire sociale (Bourdieu \& De Saint-Martin, 1975) qui elle aussi emprunte bien souvent des chemins sexués (Fassa, 2013 ; Bataille, 2014). Or, et comme le montrent Mosconi (1999, 2001, 2004) et Duru-Bellat (1995, 2017), leurs points de vue et leurs pratiques en tant qu'agents socialisateurs ont souvent été sous-estimés dans la construction de croyances et d'attitudes vis-à-vis des problématiques de genre.

En effet, comme l'explique Mosconi (1999), les relations enseignant.e-s/élèves prennent part à la construction d'identités sexuées, lesquelles contribuent à des parcours scolaires, académiques ou professionnels différents. Des traitements différenciés (quantitativement et qualitativement, Duru-Bellat, 1994, 2004) sont réservés aux deux sexes, les informant insidieusement des places qu'ils et elles doivent occuper ou viser ; ils construisent un curriculum caché (Mosconi, 2004) ayant des conséquences tant structurelles (au niveau sociétal) qu’individuelles (sur le vécu des élèves). Les

3. Précisons toutefois que ce lien n'est pas unidirectionnel : en effet, les divisons sexuées de l'orientation scolaire et professionnelle sont à leur tour une conséquence d'un monde du travail sexué. Ainsi, la relation entre ce dernier et les orientations des personnes en formation s'avère être bidirectionnelle, un facteur influençant l'autre et vice-versa.

4. soit l'ensemble de savoirs, compétences, mais également représentations et croyances qui s'acquièrent à l'école, sans qu'ils fassent partie du programme scolaire officiel. 
attitudes et les représentations des agents de socialisation que sont les enseignant.e.s contribuent à la mise en place d'une socialisation différentielle entre les sexes et au maintien des inégalités entre filles et garçons.

Dans ce cadre, il semble particulièrement intéressant d'étudier l'attitude et les représentations des enseignant.e.s des écoles professionnelles concernant le sexisme, car elles et ils sont appelé.e.s à jouer un rôle régulateur afin de développer "l'égalité effective entre les sexes" (Assemblée fédérale de la Confédération suisse, 2018). Héritant de choix de formations sexués de la part des apprenti·e.s, ces agents de socialisation doivent assumer des postures délicates vis-à-vis d'un public qui répond déjà de manière importante à des critères de ségrégation genrée (Fassa, 2016), filles et garçons ayant en majorité déjà opté pour des métiers typiquement associés à leur sexe (Guilley \& al., 2014).

Cette posture s'avère d'autant plus difficile que les enseignant.e.s ont aussi été socialisé.e.s de façon différentielle et qu'elles et ils ont suivi des parcours de formation et d'emploi sexués. De manière générale, les enseignantes sont moins nombreuses que leurs homologues masculins dans la filière de la FPI, mais elles sont en général plus diplômé.e.s qu'eux, dont une proportion importante a suivi un parcours de formation marqué par une insertion précoce dans l'emploi. Elles et ils entretiennent de ce fait des rapports différents à leurs publics, aux savoirs qu'ils et elles dispensent (Fassa $\&$ al., 2019) et comme nous allons le voir, aux stéréotypes et représentations sexistes.

S’intéresser aux croyances et aux représentations de ces enseignant.e.s semble donc important pour éclairer les dynamiques de genre dans la FPI et mieux comprendre les enjeux liés à un marché du travail fortement sexué. Flamigni \& al. (2013) montrent, à l'occasion de leur étude sur les représentations des enseignant.e.s en formation professionnelle vis-à-vis des pionnier.ère-s, que les enseignant.e.s professionnel.le.s orientent, de manière plus ou moins directe, la vision que les jeunes ont des métiers, de leurs propres identités professionnelles et de celles d'individus sexués dans le contexte du travail. Ces auteures concluent qu'en tant que co-responsables de l'éducation et de la formation des jeunes, ces enseignant.e.s ne seraient pas assez formé.e.s pour faire face aux défis de l'égalité et aux difficultés de la mixité sexuée en formation professionnelle (Flamigni \& al., 2013). 
Si, selon Mosconi (1999, 2001, 2004), ne pas adopter de pratiques visant à construire l'égalité signifie participer de fait au maintien et à la reproduction d'un ordre inégal, encore faut-il avoir conscience des inégalités liées au genre pour se former à des pratiques équitables. En endossant ce point de vue, notre contribution vise à discerner quels sont les groupes, parmi les enseignant.e.s de la FPI, qui se sont le plus nettement départis des représentations sexistes marquant les manières dont les rôles féminins et masculins sont encore conçus.

Réfléchir aux attitudes, présentes et manquantes, des enseignant·e-s semble de ce fait intéressant pour comprendre quel recul ils et elles prennent avec ce que Swim \& al. (1995) désignent comme du sexisme traditionnel ou moderne, cette réflexivité sur les rapports sociaux de sexe pouvant avoir un impact sur les pratiques professionnelles, notamment par l'adhésion ou pas à une forme de curriculum caché sexué (Mosconi, 2004). Il devient ainsi tout à fait nécessaire de comprendre quelles sont les croyances des membres du corps enseignant en ce domaine et de préciser comment ils se situent face au sexisme, soit face à des "attitudes discriminatoires basées sur le sexe " (Dictionnaire Larousse en ligne, s.d.).

\section{Méthode et population : des enseignant.e.s questionné·e.s selon des échelles de sexisme}

Une manière d'appréhender et de traiter les croyances liées au genre est proposée par Swim \& al. (1995) : il s'agit d'une échelle développée afin de mesurer ce que les auteurs ont appelé, d'une part, le sexisme traditionnel, soit les stéréotypes qui considèrent les femmes comme moins compétentes que les hommes et d'autre part, le sexisme moderne, une forme de sexisme plus subtile qui se définit "par la nonreconnaissance de la discrimination sexiste qui persiste dans notre société, par l'hostilité vis-à-vis des revendications d'égalité des chances et par le manque de soutien envers les politiques conçues pour aider des femmes" (Guilley \& al., op. cit., p. 61). Bien que l'échelle de Swim \& al. (1995) date de plus de vingt ans, la situation des femmes helvétiques reste façonnée par un régime de genre traditionnel, ce qui en justifie l'utilisation dans ce contexte spécifique (Roux, Gianettoni, \& Perrin, 2007). En raison du contexte de l'enquête et des possibilités de comparer les réponses obtenues ici avec les travaux antérieurs réalisés auprès d'enseignant.e.s de la même région (Fassa, 2014), l'usage de cette échelle particulière a été jugé plus pertinent que celles qui concernent le sexisme bienveillant $v$ s le sexisme hostile (Glick \& Fiske, 1996) ou la dominance sociale (Sidanius \& Pratto, 1999).

Cette échelle a été au cœur des analyses dans le cadre de la recherche PROFID, qui visait à investiguer les identités des enseignant.e.s de la formation professionnelle du 
canton de Vaud et leurs effets sur la transmission des savoirs aux apprenti.e.s (Fassa \& al., 2019).

Le Canton de Vaud se situe dans la partie francophone de la Suisse. Selon les données les plus récentes de l'Office Fédéral de la Statistique, et comme dans les autres cantons romands, la formation professionnelle initiale y est moins prisée que dans les cantons suisses alémaniques (à titre d'exemple, $36.4 \%$ des choix de formation dans le canton de Genève ; 47.2 \% dans le canton de Vaud et $83.8 \%$ dans le canton de Glaris, la moyenne nationale s'établissant à $67.7 \%$ en 2019 - OFS, sdl 2020). En ce qui concerne plus spécifiquement les enseignant.e.s de la filière postobligatoire de la formation professionnelle, les cantons romands y enregistrent la plus forte proportion de femmes (46.3 \% dans le canton de Genève, $42.3 \%$ dans le canton de Vaud et $34.8 \%$ dans celui de Glaris, alors que la moyenne nationale se situe à $41.5 \%$ ). Ces différents éléments, de même que les priorités des programmes de législature du gouvernement vaudois ${ }^{5}$ laissent à penser que le Canton de Vaud poursuit plutôt une politique égalitaire en regard de certains des cantons alémaniques, dans lesquels la population a parfois demandé, par voie de référendum populaire ou parlementaire, que les plans d'études n'incluent pas les questions liées à l'égalité entre les sexes (Fassa, 2016). Cette affirmation ne devrait toutefois pas être interprétée de façon trop optimiste, car les programmes de la scolarité obligatoire romande (PER, 2010 - régulièrement mis à jour en ligne - dernière consultation le 15 mai 2020) ne mentionnent les questions de genre que très ponctuellement, soit en relation avec l'objectif d'apprentissage de la formation générale : "Reconnaître l'altérité et la situer dans son contexte culturel et social» (PER, 2010, p. 22).

Menée sur mandat du Département de la Formation, de la Jeunesse et de la Culture (DFJ - équivalent du ministère de l'Éducation), l'enquête s'adressait à l'ensemble des enseignant.e.s travaillant dans les treize établissements de la formation professionnelle vaudoise qui dépendent de ce ministère. Tant du fait de la sexuation de la formation professionnelle que de la volonté affirmée des autorités d'y construire une "égalité effective ", cette recherche a inclus une batterie de questions sur les visions de l'égalité et les stéréotypes de genre.

5. Les programmes de législature du gouvernement vaudois 2012-2017 (Conseil d'État du canton de Vaud, 2012) et 2017-2022 (Conseil d'État du canton de Vaud, 2017) mettent en évidence l'égalité entre les sexes comme un des éléments majeurs de l'un de leurs quatre objectifs ("Intégration de chacun dans la société et le monde du travail »), puisque dans les deux cas, il y est stipulé, dans les premières lignes, qu' "il est essentiel, pour la cohésion du canton, que chacun puisse trouver un travail lui permettant de subvenir à ses besoins. Pour cela, il faut une économie diversifiée, et les conditions-cadres permettant notamment aux parents de concilier vie familiale et professionnelle, ainsi que la promotion des principes d'égalité des droits et d'égalité des chances, notamment entre les hommes et les femmes. " (p. 24, pour Conseil D'État du canton de Vaud, 2012 et p. 45 pour Conseil d'État du canton de Vaud, 2017). 
Les données mobilisées dans le cadre de cet article sont essentiellement les réponses aux sections d'un questionnaire plus vaste ${ }^{6}$ - les résultats indiqués par la suite se concentrant sur la question du sexisme et de son contraire, la propension à l'égalité des sexes. L'échantillon mobilisé ici prend en compte uniquement les enseignant.e.s ayant complété la partie de l'enquête PROFID sur les échelles de sexisme moderne $(\mathrm{N}=279 ; 38.71 \%$ de femmes $)$ et traditionnel $(\mathrm{N}=271 ; 40.96 \%$ de femmes $)$. Lâge moyen est de 46.63 ans $(S=9.62)$.

Les variables indépendantes considérées sont (a) le sexe ( 1 = féminin ou 2 = masculin), (b) les branches enseignées (quatre : disciplines académiques, culture générale, théorie professionnelle et pratique professionnelle) et (c) le statut des enseignant.e.s (en cinq modalités : maître-sse d'enseignement professionnel I, maître-sse d'enseignement professionnel II, maître-sse d'enseignement professionnel III, maître-sse d'enseignement postobligatoire et chargé.e de cours $-\mathrm{CDC}^{7}$ ). Le recours à ces deux dernières variables s'est avéré nécessaire tant le système est complexe dans la réalité. En effet, aussi bien le statut que le type de branches enseignées sont théoriquement à corréler avec la formation initiale et pédagogique des enseignant.e.s, mais nous avons constaté, lors des analyses, que ce n'est pas toujours le cas, loin s'en faut.

Les différents statuts correspondent aux titres pédagogiques ou académiques obtenus pour pouvoir enseigner. En ce sens, les maître-sse.s d'enseignement professionnel I (MEP-I) et II (MEP-II) ont effectué des formations professionnelles supérieures, alors que les maître.sse.s d'enseignement professionnel III (MEP-III) et les maître.sse.s d'enseignement postobligatoire (ME-PO) possèdent un diplôme de niveau Master délivré par une haute école. Ces enseignant.e.s ne perçoivent pas de salaires similaires, les titres des hautes écoles universitaires offrant de meilleures rémunérations à leurs porteur-euse.s (maître-sse du postobligatoire) que les titres des hautes écoles spécialisées (maître-sse d'enseignement professionnel III), eux-mêmes offrant une rétribution supérieure à celle obtenue à l'issue d'une formation professionnelle. Notons également que les premier.ère.s peuvent théoriquement se présenter lors des appels d'offre pour des postes dans le secondaire postobligatoire généraliste, l'équivalent des lycées généraux en France, contrairement aux second.e.s.

6. 482 enseignant.e.s ont participé à cette enquête, dont $37.8 \%$ étaient des femmes.

7. Dans le cadre de cette communication, nous n'avons pas pu effectuer d'analyses spécifiques pour les chargé.e.s de cours, soit les personnes embauchées pour une durée déterminée ou pour des cours spécifiques à chacun des métiers. Ceci s'explique par le fait qu'elles et ils n'enseignent souvent que quelques heures de théorie ou pratique professionnelles en sus de leurs activités dans leurs métiers et ne disposent donc pas nécessairement d'un diplôme pédagogique. Par ailleurs, et contrairement aux autres catégories d'enseignant·e·s, leur salaire n'est en général pas annualisé, leurs interventions dans les classes pouvant parfois même s'apparenter à des mandats de courte durée. Leur participation à la formation diffère en outre selon les domaines professionnels considérés. 
La variable " type de branches enseignées » visait, quant à elle, initialement, à préciser les rapports à différents types de savoirs, savoir-faire et savoir-être, mais elle s'est aussi avérée utile pour rendre compte, de manière indirecte, des différents parcours de formation des enseignant.e.s. En effet, les branches académiques et la culture générale sont normalement enseignées par des personnes ayant suivi des études dans des hautes écoles universitaires (université ou écoles polytechniques, soit le niveau de formation tertiaire), lorsqu'elles permettent l'obtention d'un titre de Maturité professionnelle, seul titre qui permet aux apprenti.e.s de rejoindre les filières des hautes écoles spécialisées. Les personnes qui enseignent les disciplines de théorie et/ou pratique professionnelle sont, quant à elles, majoritairement issues des formations professionnelles supérieures (FPS). Connaître les disciplines enseignées a en outre permis de nuancer une hiérarchie statutaire qui n'est pas toujours très claire dans la réalité : un " jeu " existe en effet dans les établissements, qui permet aux directions d'atténuer les différences justifiant la diversité des statuts et des activités professionnelles. Cette connaissance a ainsi rendu compte des manières dont la durée de la formation et la filière suivie (formation professionnelle, hautes écoles spécialisées et universités), ont été traduites dans les établissements, les charges effectives attribuées aux enseignant.e.s dépendant en partie des décisions des directions d'établissement. Ainsi, normalement, les maître-sse.s d'enseignement professionnel I doivent enseigner un plus grand nombre d'heures que leurs collègues, leurs activités prenant avant tout place dans des ateliers d'application ; cependant, l'enseignement de disciplines théoriques qui leur est parfois proposé par leurs directions - ou par l'organisation des formations autour de la réalisation de projets - a pour effet de diminuer en partie leur charge horaire et de leur permettre de participer à la formation des apprenti.e.s autrement que par la seule transmission des savoir-faire de métier. Certaines disciplines académiques, comme les langues étrangères et les mathématiques ou la physique, sont par ailleurs parfois considérées comme des enseignements de métier ; dès lors, une partie des enseignant.e.s qui dispensent de tels enseignements ne bénéficient pas du statut de maître-sse.s d'enseignements postobligatoires, alors que leurs études universitaires auraient dû leur garantir ce statut. 
Le Tableau 1 synthétise les éléments qui concernent le statut et les branches enseignées tels qu'ils apparaissent dans diverses directives du ministère en charge de l'Éducation et de la Formation et dans celles du service du personnel de la région étudiée.

\section{Tableau 1. Caractéristiques des parcours de formation et situation dans le métier des enseignant.e.s statutaires, en Suisse romande}

\begin{tabular}{|c|c|c|c|c|c|}
\hline $\begin{array}{l}\text { Filière de } \\
\text { formation } \\
\text { suivie }\end{array}$ & $\begin{array}{c}\text { Statut } \\
\text { (proportion de } \\
\text { femmes) }\end{array}$ & Parcours de formation & $\begin{array}{c}\text { Classe salariale et } \\
\text { salaire initial }\end{array}$ & $\begin{array}{l}\text { Branches } \\
\text { enseignées }\end{array}$ & $\begin{array}{l}\text { Nb. de périodes } \\
\text { hebdomadaires } \\
\text { d'enseignement }\end{array}$ \\
\hline \multirow[t]{2}{*}{$\begin{array}{l}\text { Formations } \\
\text { professionnelles } \\
\text { supérieures }\end{array}$} & $\begin{array}{l}\text { MEP-I } \\
(33.6 \%)\end{array}$ & $\begin{array}{l}\text { Apprentissage + ES (Maîtrise } \\
\text { ou diplômeS professionnels) }\end{array}$ & $\begin{array}{c}\text { Classe } 10 \\
\text { CHF } 80^{\prime} 278 .- \text { (env. } \\
76^{\prime} 000 € \text { ) }\end{array}$ & $\begin{array}{c}\text { Pratique } \\
\text { professionnelle }\end{array}$ & 37,5 \\
\hline & $\begin{array}{l}\text { MEP-II } \\
(26.2 \%)\end{array}$ & $\begin{array}{c}\text { Apprentissage + maturité } \\
\text { professionnelle + Bachelor } \\
\text { HES }\end{array}$ & $\begin{array}{l}\text { Classe } 11 \\
\text { CHF } 87^{\prime} 174 \text { (env. } \\
83000 € \text { ) }\end{array}$ & $\begin{array}{c}\text { Théorie } \\
\text { professionnelle }\end{array}$ & 25 \\
\hline \multirow{2}{*}{$\begin{array}{l}\text { Formation } \\
\text { dans les } \\
\text { Hautes écoles } \\
\text { spécialisées }\end{array}$} & \multirow[t]{2}{*}{$\begin{array}{l}\text { MEP-III } \\
(52.7 \%)\end{array}$} & $\begin{array}{l}\text { Apprentissage + maturité } \\
\text { professionnelle } \\
+ \text { Master HES (classe 11) }\end{array}$ & $\begin{array}{l}\text { Classe } 11 \\
\text { CHF } 87^{\prime} 174 .- \text { (env. } \\
83000 € \text { ) }\end{array}$ & $\begin{array}{c}\text { Théorie } \\
\text { professionnelle }\end{array}$ & \multirow[t]{2}{*}{25} \\
\hline & & + Master HEU (classe 12) & $\begin{array}{l}\text { Classe } 12 \\
\text { CHF } 94^{\prime} 932 .- \text { (env. } \\
90000 € \text { ) }\end{array}$ & $\begin{array}{l}\text { Disciplines } \\
\text { académiques }\end{array}$ & \\
\hline $\begin{array}{l}\text { Formation } \\
\text { dans les } \\
\text { Hautes écoles } \\
\text { universitaires } \\
\text { et les Écoles } \\
\text { polytechniques } \\
\text { fédérales }\end{array}$ & $\begin{array}{l}\text { ME-P0 } \\
(56.7 \%)\end{array}$ & $\begin{array}{c}\text { Maturité académique } \\
+ \text { Master HEU (disciplinaire) }\end{array}$ & $\begin{array}{c}\text { Classe } 12 \\
\text { CHF } 94^{\prime} 932 \text { (env. } \\
90000 € \text { ) }\end{array}$ & $\begin{array}{l}\text { Disciplines acadé- } \\
\text { miques et Culture } \\
\text { générale }\end{array}$ & 25 \\
\hline
\end{tabular}

Note 1 : Les zones grisées sont celles dans lesquelles nous avons constaté des recouvrements qui ne correspondent pas aux statuts.

Note 2 : MEP : Maître-sse d'enseignement professionnel ; ME-P : Maître-sse d'enseignement postobligatoire ; ES : École supérieure ; HES : Haute école spécialisée ; HEU : Haute école universitaire.

Lecture : Les chiffres concernant les salaires indiqués sont à contextualiser. En effet, le salaire médian en Suisse, en 2018, correspondait à 6'538 - (soit env. $6206 €$; OFS, 2020), et ceux inscrits ci-dessus sont calculés sur treize mois. Ainsi, un.e enseignant.e de la classe salariale 10 gagne $6^{\prime} 175$ - par mois (env. $5860 €$ ), ce chiffre se situant au-dessous du salaire médian helvétique, alors qu'un·e enseignant·e en classe salariale 12 obtient un salaire de 7'302 par mois (env. $6930 €)$.

Source : auteures. 
Les variables dépendantes sont quant à elles des affirmations reprises des échelles de sexisme traditionnel et moderne de Swim \& al. (1995). À partir de huit items reflétant le sexisme moderne et quinze pour le sexisme traditionnel (cf. Annexe électronique), deux nouvelles variables ont été créées (sexisme moderne : $\alpha=.81 ; \mathrm{M}=4.34$; $S=1.21$; sexisme traditionnel $: \alpha=.76 ; M=5.36 ; S=.79)$. Les deux échelles ont des scores allant de $1=$ rejet de l'égalité, jusqu'à $7=$ soutien à l'égalité ${ }^{8}$. Ainsi, pour pouvoir comparer les réponses des différents groupes aux échelles de sexisme moderne et traditionnel, des t-tests et analyses des variances ANOVA $^{9}$ ont été effectués.

\section{Un soutien à l'égalité différent 3 suivant les profils des enseignant.e.s}

Les analyses sur les moyennes aux échelles de sexisme des enseignant.e.s de la formation professionnelle vaudoise mettent en évidence que, malgré un rejet global relativement marqué des affirmations sexistes (surtout s'agissant du sexisme traditionnel), des différences existent au sein du corps enseignant.

Globalement, les participant.e.s à l'enquête semblent rejeter davantage le sexisme traditionnel que moderne, ce dernier rendant compte d'affirmations plus subtiles en termes d'égalité entre les femmes et les hommes.

Plus précisément, les femmes manifestent un soutien plus marqué vis-à-vis de l'égalité se traduisant par des affirmations telles que "Les femmes n’obtiennent souvent pas de bons postes à cause de la discrimination sexuelle " ou "Les hommes devraient soccuper davantage des enfants que ce n'est le cas actuellement" (cf. Annexe électronique). Les enseignant·e.s de culture générale (et des disciplines académiques) rejettent aussi plus fortement les propos sexistes que leurs collègues des branches professionnelles (tant de théorie que de pratique). Finalement, en comparant les différents statuts professionnels, on montrera que les ME-PO manifestent un désaccord plus net envers les échelles de sexisme que les MEP-I et II.

8. Les scores aux échelles ont été « inversés » afin de mettre l'accent sur le soutien à l'égalité, plutôt que sur le sexisme.

9. Le t-test permet de comparer les scores moyens entre deux groupes indépendants, alors que l'analyse de variance ANOVA compare les différences au niveau des variances entre plusieurs groupes indépendants. 


\subsection{Sexisme traditionnel et sexisme moderne : deux perceptions distinctes}

Un degré de désaccord plus marqué vis-à-vis des affirmations de l'échelle de sexisme moderne (par exemple, "Les inégalités entre les femmes et les hommes ne sont plus un problème en Suisse") correspond également à un rejet plus prononcé du sexisme traditionnel ("Certaines professions ne sont simplement pas appropriées aux femmes"; cf. Annexe électronique). En effet, des analyses sur les moyennes aux échelles de sexisme (Swim \& al., 1995) ont été réalisées dans les divers groupes de comparaison. Le premier constat est que les deux échelles de sexisme moderne et traditionnel variables dépendantes - sont relativement corrélées $(r .53 ; p .01)$, ce qui coïncide avec les résultats observés par Swim \& al. (1995), soit une corrélation de $r .54$.

En moyenne, les participant.e.s ont rejeté de manière plus nette les propos liés au sexisme traditionnel que ceux liés au sexisme moderne. En effet, globalement, le score moyen de l'échelle de sexisme traditionnel révèle un désaccord plus marqué envers l'échelle de sexisme traditionnel $(N=271 ; M=5.36 ; S=.79)$ que vis-à-vis de celle de sexisme moderne $(N=279 ; M=4.34 ; S=1.21)$.

Autrement dit, les enseignant.e.s ont rejeté plus nettement des affirmations prétendant que les femmes et les hommes ne devraient pas avoir le même traitement (sexisme traditionnel), comparativement aux propos du sexisme moderne qui considèrent, par exemple, que les inégalités entre les femmes et les hommes ne sont plus un problème. Ainsi, le score moyen sur l'échelle du sexisme moderne ne dépasse que légèrement la moyenne de l'échelle ${ }^{10}$, alors que la moyenne sur l'échelle du sexisme traditionnel s'éloigne nettement du score moyen de l'échelle (situé à 4).

Cela pourrait être dû au fait que les items proposés sur l'échelle du sexisme moderne sont plus "nuancés " ${ }^{11}$ que ceux de l'échelle du sexisme traditionnel, ces derniers exprimant des propos qui justifient explicitement des discriminations.

On observe globalement un rejet des deux échelles de sexisme et par conséquent une valorisation de l'égalité, mais ces positionnements diffèrent selon les sous-groupes que les enseignant.e.s forment en raison de leur sexe, des disciplines enseignées et de leur statut.

10. L'échelle allant de 1 à 7 , la moyenne " absolue " de l'échelle se situe à 4 .

11. En effet, l'échelle de sexisme moderne a pris en compte l'évolution des discours sur l'égalité et elle propose des items qui traduisent des visions sexuées de ce que « sont » et " devraient faire/être » les femmes et les hommes aujourd'hui. 


\section{2 Différences par sexe, sur les échelles de sexisme}

Les femmes affirment un rejet plus marqué que les hommes tant envers le sexisme moderne qu'envers le sexisme traditionnel. En effet, les scores moyens obtenus par les femmes reflètent un degré de désaccord plus important avec des affirmations sexistes, et donc une sensibilité plus forte aux problématiques de l'égalité que leurs collègues hommes. C'est pourquoi la moyenne aux deux échelles est plus élevée pour les enseignantes, ces dernières soutenant de manière plus importante l'égalité entre les femmes et les hommes.

Un test-t (Student) a été effectué afin d'observer si des différences significatives au niveau des moyennes aux échelles de sexisme en fonction du sexe pouvaient être mises en évidence. Les Tableaux $\mathbf{2 a}$ et $\mathbf{b}$ indiquent que des différences statistiquement significatives ont été trouvées pour les deux échelles de sexisme.

Tableau 2a. Test-t sur les moyennes par sexe, sur l'échelles de sexisme moderne $(\mathrm{N}=\mathbf{2 7 9})$

\begin{tabular}{lcccc}
\hline & Moyenne & Ecart-type & $\begin{array}{c}\text { Degré de } \\
\text { liberté }\end{array}$ & t de Student \\
\hline Femmes $(n=108)$ & 4.80 & 1.02 & 258.24 & $5.43^{* * *}$ \\
\hline Hommes $(n=171)$ & 4.06 & 1.24 & & \\
\hline Note $: p .001^{* * *}$ & & & & \\
\hline
\end{tabular}

Tableau 2b. Test-t sur les moyennes par sexe, sur l'échelles de sexisme traditionnel $(\mathrm{N}=\mathbf{2 7 1})$

\begin{tabular}{lccc}
\hline & Moyenne & Ecart-type & $\begin{array}{c}\text { Degré de } \\
\text { liberté }\end{array}$ \\
\hline Femmes $(n=111)$ & 5.72 & .52 & 265.22 \\
Hommes $(n=160)$ & 5.11 & .85 & \\
\hline
\end{tabular}

Note $: p<.001^{* * *}$

Lecture : en ce qui concerne le sexisme moderne, la moyenne obtenue par les 108 femmes qui ont répondu à cette section du questionnaire est de 4.8 sur une échelle de Likert dont le maximum est de 7. Comme l'indique le t-test, elle est significativement différente à $\leq .001$ de celle qui a été calculée sur les réponses des 171 hommes, n'ayant obtenu qu'un score de 4.06. Ceci indique que les hommes entretiennent des croyances significativement plus marquées par le sexisme moderne que les femmes. La même tendance peut être observée pour les scores obtenus à léchelle de sexisme traditionnel, où les 111 femmes ayant répondu ont une moyenne de 5.72, soit significativement différente à $\leq .001$ par rapport à la moyenne obtenue par les hommes (5.11).

Source : auteures.

\subsection{Différences sur les échelles de sexisme, par type de branche enseignée}

Les enseignant.e.s de culture générale - mais également celles et ceux des disciplines académiques - s'opposent plus fortement aux affirmations sexistes, suivi.e.s par les collègues enseignant la théorie professionnelle. Les personnes enseignant la pratique professionnelle semblent, quant à elles, être les moins sensibles aux problématiques de l'égalité, si l'on se réfère aux moyennes des échelles de sexisme. 
En effet, la discipline enseignée reflète les parcours de formation et professionnels des enseignant.e.s et, parallèlement, elle rend aussi compte des divers domaines d'enseignement en formation professionnelle. Ainsi, une analyse des variances (ANOVA) pour quatre modalités a été effectuée : les branches dites académiques, celles de culture générale, la théorie professionnelle, ainsi que la pratique professionnelle. Une comparaison des scores moyens aux deux échelles de sexisme a donc été réalisée en tenant compte du type de branche enseignée (cf. Tableaux 3a et b).

Tableau 3a. Analyse de la variance sur l'échelle de sexisme moderne, par type de branche enseignée $(\mathrm{N}=276)$

\begin{tabular}{|l|c|c|}
\hline & Moyenne & Ecart-type \\
\hline Disciplines académiques $(n=83)$ & 4.62 & .99 \\
\hline Culture générale $(n=39)$ & 4.97 & 1.06 \\
\hline Théorie professionnelle $(n=105)$ & 4.12 & 1.23 \\
\hline Pratique professionnelle $(n=49)$ & 3.83 & 1.34 \\
\hline
\end{tabular}

Tableau 3b. Analyse de la variance sur l'échelle de sexisme traditionnel, par type de branche enseignée $(\mathrm{N}=\mathbf{2 7 0})$

\begin{tabular}{|l|c|c|}
\hline & Moyenne & Ecart-type \\
\hline Disciplines académiques $(\mathrm{n}=84)$ & 5.46 & .71 \\
\hline Culture générale $(\mathrm{n}=34)$ & 5.75 & .65 \\
\hline Théorie professionnelle $(\mathrm{n}=102)$ & 5.25 & .81 \\
\hline Pratique professionnelle $(\mathrm{n}=50)$ & 5.11 & .84 \\
\hline
\end{tabular}

Lecture : Concernant le sexisme moderne, les enseignant-e.s de culture générale soutiennent davantage l'égalité que leurs collègues de théorie professionnelle (ceci significativement à <.01) et de pratique professionnelle (le seuil de significativité étant $<.001$ ). II en est de même pour le sexisme traditionnel, où les enseignant.e.s de culture générale ont répondu significativement plus en faveur de l'égalité que leurs collègues de théorie et de pratique professionnelle.

Source : auteures.

Plus précisément, en ce qui concerne la variable sexisme moderne, le test de Levene indiquait des variances hétérogènes au sein de la variable branche ; c'est pourquoi il a fallu recourir au test non-paramétrique de Kruskal-Wallis ${ }^{12}$, test qui a mis en évidence des différences significatives entre le groupe d'enseignant.e.s de culture générale et ceux de théorie professionnelle $(p<.01)$ et de pratique professionnelle $(p<.001)$. Ces résultats pour l'échelle de sexisme moderne semblent signifier qu'en moyenne, les

12. Ce test étant l'équivalent non-paramétrique de l'ANOVA. 
enseignant·e.s de culture générale rejettent plus nettement les affirmations sexistes que leurs collègues de théorie professionnelle et de pratique professionnelle ${ }^{13}$.

Quant à l'échelle de sexisme traditionnel, l'analyse de la variance s'est avérée statistiquement significative au seuil de $1 \%\left(F_{3.266}=5.80, p<.001\right)$. Compte tenu de la présence de plusieurs groupes, un test post-hoc de Scheffé a été nécessaire afin de savoir quelles différences étaient significatives - au niveau des variances - parmi les quatre types de discipline. Les résultats montrent, comme pour l'échelle de sexisme moderne, que le groupe d'enseignant.e.s de culture générale a une moyenne significativement plus élevée que les groupes de théorie professionnelle $(p<.05)$ et de pratique professionnelle $(p<.01)$.

\subsection{Différences sur les échelles de sexisme, par statut professionnel}

Les enseignant.e.s ayant le statut de maîtres.se.s de l'enseignement postobligatoire semblent plus souvent rejeter le sexisme, si on les compare à leurs collègues de l'enseignement professionnel I et II. Ceci est surtout le cas des affirmations faisant référence à des formes de discrimination plus nuancées (le sexisme moderne), alors que pour le sexisme traditionnel, les différences dans les moyennes restent significatives, mais sont plus faibles que dans le cas des scores relatifs à l'échelle de sexisme moderne.

Autrement dit, les MEP-I ${ }^{14}$ et MEP-II sont celles et ceux qui rejettent le moins les propos sexistes des échelles de Swim \& al. (1995), alors que les ME-PO s'opposent de manière plus importante au sexisme. Il semblerait donc que ce dernier groupe soit le plus sensible aux problématiques de l'égalité des genres.

Ainsi, en effectuant une analyse de la variance (ANOVA) des différents statuts, des variations significatives des moyennes aux échelles de sexisme ont pu être mises en évidence (Sexisme moderne $: F_{4,257}=6.155 ; p<.001$. Sexisme traditionnel : $F_{4,253}$ $5.297 ; p<.001$; cf. Tableaux $4 \mathbf{a}$ et b). Afin de savoir où se situent les différences significatives parmi les cinq groupes, un test post-hoc de Scheffé a aussi été effectué. Ce dernier a permis de mettre en évidence les aspects suivants : pour l'échelle de sexisme moderne, une différence des moyennes significative existe entre le statut de MEP-I et de ME-PO $(p<.001)$, ainsi qu'entre le statut de MEP-II et de ME-PO $(p<.01)$. Des différences significatives pour l'échelle de sexisme traditionnel s'observent également entre le statut de MEP-I et de ME-PO $(p<.01)$. Une tendance à la significativité

13. Aussi, même si le score n'est pas statistiquement significatif, on observe que les enseignant.e.s des disciplines académiques ont en moyenne un score se rapprochant davantage de celui des collègues de culture générale que du score des collègues des branches professionnelles.

14. Note : MEP-I : maître-sse d'enseignement professionnel I ; MEP-II : maître-sse d'enseignement professionnel ; MEP-III : maître-sse d'enseignement professionnel III ; ME-PO : maître-sse d'enseignement postobligatoire ; CDC : chargé.e de cours. 
$(p=.052)$ est, quant à elle, présente entre le statut de MEP-II et de ME-PO. Aucune différence statistiquement significative n'a été mise en évidence dans les autres groupes pour le statut professionnel (MEP-III et CDC).

Tableau 4a. Analyse de la variance, sur l'échelle de sexisme moderne par statut professionnel $(\mathrm{N}=\mathbf{2 5 8})$

\begin{tabular}{|l|c|c|}
\hline & Moyenne & Ecart-type \\
\hline Maître·sse d'enseignement professionnel I (MEP-I; $n=87)$ & 4.01 & 1.22 \\
\hline Maître·sse d'enseignement professionnel II (MEP-II ; $\mathrm{n}=54)$ & 4.02 & 1.26 \\
\hline Maître-sse d'enseignement professionnel III (MEP-III; $\mathrm{n}=20)$ & 4.24 & 1.13 \\
\hline Maître-sse d'enseignement postobligatoire (MEP-PO; $\mathrm{n}=78)$ & 4.82 & 1.08 \\
\hline Chargé.e de cours (CDC; $\mathrm{n}=19)$ & 4.51 & 1.07 \\
\hline
\end{tabular}

Tableau 4b. Analyse de la variance, sur l'échelles de sexisme traditionnel par statut professionnel $(\mathrm{N}=254)$.

\begin{tabular}{|l|c|c|}
\hline & Moyenne & Ecart-type \\
\hline Maître-sse d'enseignement professionnel I (MEP-I; $n=85)$ & 5.14 & .83 \\
\hline Maître.sse d'enseignement professionnel II (MEP-II ; $n=59)$ & 5.24 & .79 \\
\hline Maître-sse d'enseignement professionnel III (MEP-III ; $n=18)$ & 5.61 & .78 \\
\hline Maître-Sse d'enseignement postobligatoire (MEP-PO ; $n=74)$ & 5.65 & .64 \\
\hline Chargé.e de cours (CDC; $n=18)$ & 5.29 & .90 \\
\hline
\end{tabular}

Lecture : concernant l'échelle de sexisme moderne, les enseignant·e-S MEP-I (4.01) et MEP-II (4.02) présentent une moyenne significativement inférieure à celle de leurs collègues ME-PO (4.82), ce qui signifie que ces dernier-ère-s se montrent plus soutenant·e·s vis-à-vis de l'égalité. Il en va de même pour l'échelle de sexisme traditionnel, où les enseignant·e.s MEP-I (5.14) ont une moyenne significativement inférieure aux collègues ME-P0 (5.65). Source : auteures.

\section{Discussion}

Il existe des différences - au niveau du sexe des enseignant.e.s, mais également du type de discipline enseignée et du statut professionnel - quant aux échelles de sexisme (Swim \& al., 1995) au sein du corps enseignant de la formation professionnelle vaudoise. Il est intéressant de constater que ces agents de socialisation acceptent - et probablement véhiculent - encore en partie des stéréotypes liés aux rôles des femmes et des hommes dans la société suisse. En ce sens, l'image de «l'homme gagne pain » et de la femme qui se consacre à la sphère domestique n'est pas complètement rejetée par le corps enseignant de la formation professionnelle initiale, tout comme la reconnaissance des mêmes droits aux personnes des deux sexes n'est pas tout à fait soutenue. 
Ces stéréotypes semblent par ailleurs plus vivaces dans les groupes d'enseignant.e.s dont le parcours antérieur comporte nécessairement une période de travail dans les entreprises, mais qui est aussi en général marqué par un temps scolaire plus bref ${ }^{15}$. Les stéréotypes de genre semblent donc ne pas avoir été suffisamment ébranlés durant la formation purement scolaire et ont au contraire été probablement cristallisés par la participation au monde du travail, celui-ci s'avérant toujours très sexué et ségrégué horizontalement (Borkowsky, op. cit.).

Par ailleurs, et avec la promotion de l'égalité entre les sexes, si le sexisme traditionnel (forme de sexisme manifeste ; Tougas \& al., 2005) est devenu inacceptable, le sexisme moderne - ou néo-sexisme (Tougas \& al., 1995) - continue d'avoir des effets sur l'égalité dans le monde du travail, surtout en termes d'inclusion des femmes dans des secteurs professionnels traditionnellement «masculins» (Tougas \& al., 2005).

Il est donc légitime de se demander dans quelle mesure la présence de telles croyances peut avoir une influence sur la formation des jeunes en formation professionnelle. Ainsi, le rôle des enseignant.e.s en tant qu'agents de socialisation (trop souvent encore asymétrique Duru-Bellat, 2004) est au cœur du débat relatif aux inégalités de genre dans le contexte particulier de la formation professionnelle. En effet, et comme nous l'avons signalé auparavant, les enseignant.e.s qui forment des apprenti-e.s ont la spécificité d'hériter d'élèves ayant déjà effectué des choix de formation reflétant des aspirations professionnelles fortement genrées (Gianettoni \& al., 2015), et dont la majorité ose rarement «transgresser » les normes de genre à travers des carrières atypiques (Gianettoni \& al., 2010).

Par ailleurs, les enseignant.e.s professionnel.le.s, qui peuvent jouer le rôle de modèle pour les apprenti.e.s, occupent leur fonction en ayant hérité des structures du monde du travail et en ayant été peu exposé.e.s à des formations qui portent à discuter de l'évidence - et de la naturalité - du genre. Ils et elles sont aussi peu enclin.e.s à remettre en cause les rapports sociaux de sexe. Les hommes sont ainsi beaucoup plus nombreux parmi les enseignant.e.s dans les domaines peu féminisés - tels la construction, la mécanique ou encore la technologie (Fassa, 2016) - alors que les enseignements des domaines féminisés - éducation, santé et social, mais aussi soins à la personne (Gianettoni \& al., 2010) - sont plus souvent dispensés par des femmes. Ces deux mécanismes conjugués ont pour effet que les stéréo-

15. L'accès à la voie professionnelle se fait, pour la grande majorité des personnes, à l'issue de la scolarité obligatoire. Or, dès l'entrée en apprentissage, qui se déroule principalement sous sa forme duale, un temps comparativement moindre à celui qui est consacré dans la voie générale du secondaire postobligatoire (lycées généralistes) est dédié aux connaissances générales (histoire, littérature, mathématiques et sciences de la vie par exemple). Les enseignant.e.s des branches théoriques de métier qui viennent du monde professionnel sont dans cette situation. Il reste toutefois difficile d'affirmer que la durée moindre des études en est responsable, tout comme il est impossible d'attribuer cette moindre sensibilité aux inégalités de genre exclusivement à leur insertion antérieure dans le monde des entreprises. Notons cependant que ce constat va dans le même sens que celui de Fassa, Rolle \& Storari (2014) à propos des liens entre formation initiale et sensibilité aux inégalités de genre dans l'éducation obligatoire romande. 
types de genre, tels que mis en évidence par les analyses menées, sont plus marqués au sein des enseignant·e.s dispensant les enseignements théoriques et les pratiques de métiers.

Jouant un rôle primordial dans la transmission de savoirs et connaissances formels, les enseignant.e.s sont aussi - voire surtout - les vecteurs d'un ensemble de croyances et représentations plus informelles (qui rentrent dans le curriculum caché ; Mosconi, 2004). Parmi ces éléments figurent aussi ceux qui ont trait aux rôles sociaux des femmes et des hommes et aux opportunités professionnelles qui s'offrent aux deux sexes. Or, au vu des résultats aux échelles de sexisme, la nécessité de sensibiliser davantage le corps enseignant de la formation professionnelle semble s'imposer si les Autorités cantonales veulent répondre aux injonctions de la Loi sur la formation professionnelle en termes d'égalité des sexes : "La présente loi encourage et développe l'égalité des chances de formation sur le plan social et à l'échelle régionale, l'égalité effective entre les sexes de même que l'élimination des inégalités qui frappent les personnes handicapées dans la formation professionnelle " (Assemblée fédérale de la Confédération suisse, 2018, Art. 3c).

Ceci semble important, d'une part, pour que la croyance en une égalité entre filles et garçons en formation ne soit plus considérée comme déjà acquise (Guilley \& al., op. cit.) et qu'elle se dessine comme un objectif à atteindre impliquant des actions; d'autre part, pour que le corps enseignant se rende compte des stéréotypes et des représentations de genre qu'il contribue à véhiculer plus ou moins inconsciemment lors de la transmission en classe (Flamigni \& Pfister Giauque, op. cit.). Plus précisément, les enseignant.e.s en formation professionnelle - mais également les institutions publiques, les familles, et les personnes formatrices en entreprise - pourraient améliorer leurs pratiques formatives si ils et elles étaient sensibilisé.e.s à leur part de responsabilité dans la transmission et la perpétuation des représentations et stéréotypes sexués, ce qui pourrait notamment les motiver à s'engager davantage dans des actions concrètes en faveur de l'égalité.

\section{Conclusion}

À partir d'un état des lieux de la formation professionnelle en Suisse, à l'aune du genre, ainsi que des travaux qui considèrent les enseignant.e.s comme des agents de socialisation pour les jeunes (tant pour leur éducation que pour leur formation), les stéréotypes de genre d'un échantillon d'enseignant.e.s en formation professionnelle vaudoise ont été analysés. Plus précisément, l'intérêt de ce travail était d'investiguer le point de vue des enseignant.e.s quant à leurs représentations plus ou moins égalitaires. Ceci en sachant que les valeurs, stéréotypes et normes du corps enseignant sont liés à une socialisation différentielle des sexes au monde professionnel, qui est aussi tributaire de la formation initiale (filière professionnelle ou scolaire et universitaire) et de la participation aux mondes des métiers.

Ainsi, les analyses sur les moyennes aux échelles de sexisme, traditionnel et moderne, ont mis en avant des différences significatives entre les femmes et les hommes enseignant.e.s, mais également entre les différents types de disciplines enseignées et entre les statuts pro- 
fessionnels. S’intéresser aux agents de socialisation que sont les enseignant.e.s en formation professionnelle en privilégiant une perspective de genre s'avère une piste intéressante pour la recherche et pour l'action éducative.

En ce qui concerne la recherche, elle a permis, d'une part, de confirmer, pour le cas vaudois, les résultats d'autres chercheur.e.s : le lien entre sexisme moderne et traditionnel, ce qui corrobore les résultats mis en avant par Swim \& al. (1995), et la tendance à rejeter plus intensément le sexisme traditionnel que sa forme moderne, comme Barreto \& Ellemers (2005) l'ont montré. Notre travail a par ailleurs permis de mettre en lumière certains aspects liés aux opinions et représentations du corps enseignant de cette filière qui n'avaient pas encore été explorées dans le contexte helvétique. En effet, à notre connaissance, les travaux suisses sur les enseignant.e.s en FPI concernent principalement leurs motivations et les reconversions en direction de l'enseignement (Baumeler \& Berger 2017 ; Berger, 2018 ; Berger \& D’Ascoli, 2012), mais aucun n'a traité de manière statistique de leurs attitudes vis-à-vis de l'égalité entre les sexes. Il serait néanmoins intéressant de pouvoir approfondir nos résultats, notamment en poursuivant l'enquête sur les parcours de formation des enseignant.e.s de la FPI. Il s'agirait de préciser les causes des nuances que nous avons constatées au sein des différents groupes enseignants, quant à leurs conceptions de l'égalité de genre.

Du côté de l'action éducative, ces résultats conduisent à affirmer qu'une formation spécifique à l'égalité reste nécessaire afin de répondre aux autorités politiques cantonales désireuses de lutter contre les stéréotypes de genre dans les métiers. Notre travail révèle ainsi qu'il faut encore sensibiliser les enseignant.e.s à cette thématique afin qu'ils et elles puissent encourager les choix atypiques de certain.e.s élèves. Toutefois, il ne permet pas d'affirmer que l'ensemble des stéréotypes et représentations genrés des enseignant·e.s marquent leurs pratiques professionnelles d'une manière telle qu'ils se transmettent à leurs élèves ; les données disponibles dans le cadre de l'enquête PROFID ne permettant pas, en effet, de répondre à cette question.

Aussi, il serait fort intéressant, à la suite de ce travail, d'explorer - idéalement avec un échantillon plus conséquent - le lien potentiel entre les représentations et croyances personnelles du corps enseignant et leur transmission dans le contexte de formation à l'école. Plus précisément, ce travail pourrait servir d'appui pour investiguer, de façon plus systématique que n’ont pu le faire Flamigni \& Pfister Giauque (op. cit.) - par le biais notamment d'observations pendant des cours en école professionnelle -, si et comment les enseignant.e.s de la formation professionnelle transmettent, de manière consciente ou pas, leur point de vue sur l'égalité. Étudier les curricula cachés des enseignant·e.s - en tant qu'agents de socialisation - permettrait également d'appréhender de façon plus informée la vision que les jeunes développent du monde du travail du point de vue du genre. 


\section{Bibliographie}

Assemblée fédérale de la Confédération suisse (2018), Loi fédérale sur la formation professionnelle (LFPr). Accès : https://www.admin.ch/opc/fr/classified-compilation/20001860/201801010000/412.10.pdf

Barreto M. \& Ellemers N. (2005), “The perils of political correctness: Men's and women's responses to old-fashioned and modern sexist views", Social psychology quarterly, 68(1), pp. 75-88.

Bataille P. (2014), Des cheminements sur la voie royale. Une analyse sociologique des parcours de vie des normalien.ne.s de Saint-Cloud, Fontenay-aux-Roses et Lyon (1981-1987), thèse de doctorat en sociologie, Université de Lausanne.

Baumeler C. \& Berger J.-L. (2017), Besoin et recrutement d'enseignant-e-s qualifié-e-s pour la formation professionnelle initiale. SRFP Newsletter, 3.

Berger J.-L. (2018), "Motivations et identité professionnelle des enseignants en école professionnelle ", L'Éducateur, Numéro spécial, pp. 18-19.

Berger J.-L. \& D’Ascoli Y. (2012), Enseigner en formation professionnelle : quels sont les facteurs qui influencent ce choix ?, Lausanne, Institut fédéral des hautes études en formation professionnelle IFFP.

Borkowsky A. (2000), „Frauen und Männer in der Berufsbildung SchweizSchweizerische”, Zeitschrift für Bildungswissenschaften, 22(2), pp. 279-294.

Bourdieu P. \& De Saint-Martin M. (1975), « Les catégories de l'entendement professoral», Actes de la recherche en sciences sociales, 3, pp. 68-93.

Bressoux P. (1994), " Note de synthèse : les recherches sur les effets-écoles et les effetsmaîtres ", Revue française de pédagogie, 108(1), pp. 91-137.

Bressoux P. (2009), « Des contextes scolaires inégaux : effet-établissement, effet-classe et effets du groupe de pairs ", in Duru-Bellat M. \& Van Zanten A. (Eds.), Sociologie du système éducatif. Les inégalités scolaires, Paris, PUF, pp. 131-148.

Bressoux P. \& Lima L. (2011), «La place de l'évaluation dans les politiques éducatives : le cas de la taille des classes à l'école primaire en France ", in Felouzis G. \& Hanhar S. (Eds.), Gouverner l'éducation par les nombres - Raisons éducatives Bruxelles, De Boeck, pp. 99-124.

Buchs H. \& Müller B. (2016), «L'offre d'emplois conditionne la qualité de l'intégration dans le marché du travail Suisse. Une comparaison formation duale / formation en école ", Formation Emploi, 133, pp. 55-75.

Conseil d'État du canton de Vaud (2012), Programme de législature 2012-2017. Accès https://www.vd.ch/fileadmin/user_upload/organisation/ce/fichiers_pdf/programmelegislature-2012-2017_web.pdf 
Conseil d'État du canton de Vaud (2017), Programme de législature 2017-2022. Accès https://www.vd.ch/fileadmin/user_upload/organisation/ce/fichiers_pdf/progleg_2017-2022-final-numerique.pdf

CSRE (2014), Le système éducatif suisse. L'éducation en suisse, Rapport 2014 (CSRE). Aarau Ed.

Dictionnaire Larousse en ligne (s.d.)., Sexisme. Accès : http://www.larousse.fr/ dictionnaires/francais/sexisme/72461?q=sexisme\#71652

Duru-Bellat M. (1994), "Filles et garçons à l'école, approches sociologiques et psychosociales ", Revue française de pédagogie, 109(1), pp. 111-141.

Duru-Bellat M. (2004), «Ecole de garçons et école de filles... Diversité-ville, école intégration ", Revue Plurielles, 138, pp. 65-72.

Duru-Bellat M. (2010), "La mixité à l'école et dans la vie, une thématique aux enjeux scientifiques forts et ouverts ", Revue française de pédagogie, 2(171), pp. 9-13.

Duru-Bellat M. (2017), La Tyrannie du genre, Paris, Presses de Sciences Po.

Duru-Bellat M. \& Leroy-Audouin C. (1990), «Les pratiques pédagogiques au CP : structures et incidences sur les acquisitions des élèves ", Revue française de pédagogie, (93), pp. 5-15.

Duru-Bellat M. (1995), "Filles et garçons à l'école, approches sociologiques et psychosociales. Deuxième partie : la construction scolaire des différences entre les sexes ", Revue française de pédagogie, janvier-mars, 110, pp. 75-109.

Falcon J. (2016), «Les limites du culte de la formation professionnelle : comment le système éducatif suisse reproduit les inégalités sociales ", Formation Emploi, 133, pp. 35-53.

Fassa F. (2013), "Choix professionnels, orientations temporelles et scripts sexués des professions ", LIVES Working Papers. Special edition: Genre et orientation scolaire et professionnelle, pp. 4-38.

Fassa F. (2014), "Éducation à l'égalité, perceptions et pratiques enseignant.e.s ", in Collet I. \& Dayer C. (Eds.), Que nous apprend le genre? Enjeux et paradoxes de l'éducation-formation, Bruxelles, De Boeck, pp. 69-86.

Fassa F. (2016), Filles et garçons face à la formation. Les défis de l'égalité, Lausanne, PPUR.

Fassa F. \& Naef C. (2015), «Deux interventions en faveur de l'égalité dans l'école obligatoire : les raisons d'un devenir contrasté ", Revue Suisse Des Sciences de l'Éducation, 37(2), pp. 267-284.

Fassa F., Dubois S., Wenger M. \& Ceppi J. (2019), Les identités professionnelles des enseignant.e.s de la Formation Professionnelle Initiale vaudoise, Lausanne. Accès https:// serval.unil.ch/notice/serval:BIB_6D8B515C1BC8 
Fassa F., Fueger H., Lamamra N., Chaponnière M. \& Ollagnier E. (2010), « Éducation et formation: enjeux de genre ", Nouvelles questions féministes, 29(2), pp. 4-16.

Fassa F., Rolle V. \& Storari C. (2014), « Politiques de l'égalité à l'école obligatoire. Des ambivalences qui diluent les rapports sociaux de sexe ", Revue suisse de sociologie, 40(2).

Fazekas M. \& Field S. (2013), "A Skills beyond School Review of Switzerland, OECD”, Reviews of Vocational Education and Training, Paris, OECD Publishing.

Flamigni E. \& Pfister Giauque B. (2014), «La mobilité de genre à l'épreuve du discours enseignant ", Nouvelles Questions Féministes, 33(1), pp. 49-63.

Flamigni E., Caprani I. \& Pfister Giauque B. (2013), «Égalité et non-mixité dans la formation professionnelle : un chantier à investir ", Formation et pratiques d'enseignement en questions - Formation à la profession enseignante : des savoirs en tout genre, 16, pp. 129-142.

Gianettoni L. \& Guilley E. (2016), "Sexism and the gendering of professional aspirations”, in Faniko K., Lorenzi-Cioldi F., Sarrasin O. \& Mayor E., Gender and social hierarchies: perspectives from social psychology, London, Routledge, pp. 11-25.

Gianettoni L., Carvalho Arruda C., Gauthier J.-A., Gross D. \& Joye D. (2015), "Aspirations professionnelles des jeunes en Suisse : rôles sexués et conciliation travail/ famille ", Social Change in Switzerland, 3, pp. 1-9.

Gianettoni L., Simon-Vermot P. \& Gauthier J. A. (2010), «Orientations professionnelles atypiques : transgression des normes de genre et effets identitaires ", Revue française de pédagogie, (4), pp. 41-50.

Glick P., \& Fiske S. T. (1996), "The ambivalent sexism inventory: Differentiating hostile and benevolent sexism", Journal of personality and social psychology, 70(3), pp. 491-512.

Guilley E., Carvalho Arruda C., Gauthier J. A., Gianettoni L., Gross D., Joye D. \& Müller K. (2014), Maçonne ou avocate : rupture ou reproduction sociale. Une enquête sur les aspirations professionnelles des jeunes en Suisse aujourd'hui, Genève, Lausanne, SRED, LINES.

Heiniger M. \& Imdorf C. (2018), “The role of vocational education in the transmission of gender segregation from education to employment: Switzerland and Bulgaria compared", Journal for labour market research, 52(1), pp. 1-21.

Lamamra N. (2016), Le genre de l'apprentissage, l'apprentissage du genre : quand les arrêts prématurés révèlent les logiques à l'oeuvre en formation professionnelle initiale, Zürich, Seismo.

Mosconi N. (1999), "Les recherches sur la socialisation différentielle des sexes à l'école ", Débats Jeunesses, 4(1), pp. 85-116. 
Mosconi N. (2001), "Comment les pratiques enseignantes fabriquent-elles de l'inégalité entre les sexes ", séminaire du Cref, Equipe "Savoirs et rapport au savoir ", mars, Paris X-Nanterre.

Mosconi N. (2004), "Effets et limites de la mixité scolaire ", Travail, genre et sociétés, 1, pp. 165-174.

OFS (2017), Choix de formation au degré secondaire II, évolution. Accès : https://www.bfs. $\mathrm{admin.ch/bfs/fr/home/statistiques/education-science/indicateurs-formation/systeme-}$ formation-suisse/degre-formation/degre-secondaire-ii/formations-degre-secondaire. assetdetail.2924593.html

OFS (2020), Enquête suisse sur la structure des salaires en 2018 : premiers résultats. Accès https://www.bfs.admin.ch/bfs/fr/home/statistiques/travail-remuneration/salairesrevenus-cout-travail/niveau-salaires-suisse.assetdetail.11927345.html

PER (2010). Formation générale. Accès https://www.plandetudes.ch/documents/10273/36907/PER_print_FG_C3_CommentGeneraux.pdf

Roux P., Gianettoni L. \& Perrin C. (2007), «L'instrumentalisation du genre : une nouvelle forme de racisme et de sexisme ", Nouvelles questions féministes, 26(2), pp. 92-108.

Sidanius J. \& Pratto F. (1999), Social dominance: an intergroup theory of social hierarchy and oppression, New Work, Cambridge University Press.

Swim J. K., Aikin K. J., Hall W. S. \& Hunter B. A. (1995), "Sexism and racism: Oldfashioned and modern prejudices", Journal of personality and social psychology, 68(2), p. 199.

Tougas F., Beaton A. M. \& Laplante J. (2005), « Le sexisme, un barrage à deux voies : la résistance à l'intégration des femmes dans un secteur traditionnellement masculin ", Les Cahiers internationaux de psychologie sociale, 3, pp. 23-32.

Tougas F., Brown R., Beaton A. M. \& Joly S. (1995), « Neosexism : plus ça change, plus c'est pareil », Personality and Social Psychology Bulletin, 21(8), pp. 842-849.

Vouillot F. (2007), "L'orientation aux prises avec le genre », Travail, genre et sociétés, 18(2), pp. 87-108. 